\title{
The Separation and Characterization of Extracellular Vesicles from Medium Conditioned by Bovine Embryos
}

\author{
Krishna Chaitanya Pavani ${ }^{1, *}$, Xiaoyuan Lin ${ }^{2}$, Joachim Hamacher ${ }^{3}$, Wim Van Den Broeck ${ }^{4}(\mathbb{D}$, \\ Liesbeth Couck ${ }^{4}$, Luc Peelman ${ }^{2}$, An Hendrix ${ }^{5,6}$ and Ann Van Soom ${ }^{1}$ (D) \\ 1 Department of Reproduction, Obstetrics and Herd Health, Faculty of Veterinary Medicine, University of \\ Ghent, Salisburylaan 133, B-9820 Merelbeke, Belgium; Ann.Vansoom@ugent.be \\ 2 Department of Nutrition, Genetics and Ethology, Faculty of Veterinary Medicine, Ghent University, \\ B-9820 Merelbeke, Belgium; linxiaoyuan23@163.com (X.L.); Luc.Peelman@ugent.be (L.P.) \\ 3 Plant Diseases and Crop Protection, Institute of Crop Science and Resource Conservation, University of \\ Bonn, Nussallee 9, 53115 Bonn, Germany; hamacher@uni-bonn.de \\ 4 Department of Morphology-Faculty of Veterinary Medicine, Ghent University, Salisburylaan 133, \\ B-9820 Merelbeke, Belgium; Wim.VandenBroeck@UGent.be (W.V.D.B.); liesbeth.couck@ugent.be (L.C.) \\ 5 Laboratory of Experimental Cancer Research, Department of Human Structure and Repair, Ghent University, \\ B-9000 Ghent, Belgium; an.hendrix@ugent.be \\ 6 Cancer Research Institute Ghent (CRIG), B-9000 Ghent, Belgium \\ * Correspondence: krishnachaitanya.pavani@ugent.be
}

Received: 31 March 2020; Accepted: 21 April 2020; Published: 22 April 2020

\begin{abstract}
Extracellular vesicles (EVs) have been identified as one of the communication mechanisms amongst embryos. They are secreted into the embryo culture medium and, as such, represent a source of novel biomarkers for identifying the quality of cells and embryos. However, only small amounts of embryo-conditioned medium are available, which represents a challenge for EV enrichment. Our aim is to assess the suitability of different EV separation methods to retrieve EVs with high specificity and sufficient efficiency. Bovine embryo-conditioned medium was subjected to differential ultracentrifugation (DU), OptiPrep ${ }^{\mathrm{TM}}$ density gradient (ODG) centrifugation, and size exclusion chromatography. Separated EVs were characterized by complementary characterization methods, including Western blot, electron microscopy, and nanoparticle tracking analysis, to assess the efficiency and specificity. OptiPrep ${ }^{\mathrm{TM}}$ density gradient centrifugation outperformed DU and SEC in terms of specificity by substantial removal of contaminating proteins such as ribonucleoprotein complexes (Argonaute-2 (AGO-2)) and lipoproteins (ApoA-I) from bovine embryo-derived EVs (density: 1.02-1.04, 1.20-1.23 g/mL, respectively). In conclusion, ODG centrifugation is the preferred method for identifying EV-enriched components and for improving our understanding of EV function in embryo quality and development.
\end{abstract}

Keywords: extracellular vesicles; isolation; protocols; ultracentrifugation; size exclusion chromatography; Optiprep $^{\mathrm{TM}}$ density gradient

\section{Introduction}

Over the last two decades, extracellular vesicles (EVs) have been recognized as a critical component of intercellular communication in both physiological and pathological conditions [1]. Interestingly, they are present in all kinds of biological fluids and contain a myriad of biomolecules, such as proteins, nucleic acids, and metabolites. Therefore EVs represent a very attractive source of potential biomarkers, particularly in the enhancement of assisted reproductive technologies (ART) in humans. 
Several sophisticated models based on multivariate parameters of embryo scoring have been designed to predict pregnancy rates in humans after in vitro fertilization and to optimize the selection of embryos in specific subgroups of patients [2]. Although these models are a step forward, they still rely on morphological observations of embryo development and do not take into account the genetic constitution of the embryo. Embryos carrying chromosomal abnormalities will have a lower chance of implantation or will lead to a higher percentage of pregnancy loss after implantation $[3,4]$.

Embryonic secretions might predict the true developmental capacity of an embryo. The embryo culture medium could, therefore, reveal important quality aspects of the embryo that could assist the selection of the most competent embryos [5]. Recently, our group has demonstrated that bovine embryos cultured in a group can release functionally active EVs with sizes ranging from 25 to $250 \mathrm{~nm}$. Embryo-derived EVs are possible autocrine embryotropins playing a crucial role in embryo development [6]. Recently, Giacomini et al. [7] have also shown that conditioned media from human embryos cultured in vitro for 3 days or up to the blastocyst stage contained EVs with a diameter of 50 to $200 \mathrm{~nm}$.

The separation of EVs still represents a critical step due to several reasons: first, the methods to isolate EVs are currently highly diverse, and second, depending on which separation method is employed, the results can be considerably different, even when starting from the same sample [8]. To date, DU is the most popular technique for the separation of EVs, which typically consists of low-speed centrifugation to remove cells and large vesicles followed by high-speed ultracentrifugation to pellet EVs [9]. This method relies on sedimentation at high speed for separating EVs from other (extra)cellular components [10,11]. Density gradient-based centrifugation, using sucrose or iodixanol (OptiPrep ${ }^{\mathrm{TM}}$ ), can be utilized to remove contaminating impurities such as non-specific argonaute proteins, and high-density lipoproteins [8]. Apart from both ultracentrifugation methods, size exclusion chromatography (SEC) is also commonly used. Size-exclusion chromatography separates EVs from other media components based on their size, whereby particles with different sizes move through the filtration column at different rates.

Our aim is to assess the suitability of different methods to separate EVs with maximum specificity and efficiency from embryo-conditioned medium. We evaluate the impact of three separation methods (differential ultracentrifugation (DU), OptiPrep ${ }^{\mathrm{TM}}$ density gradient ultracentrifugation (ODG), and size-exclusion chromatography (SEC)) on yield, purity, size, and morphology of EVs secreted by bovine embryos.

\section{Results}

A total of 1500 presumed zygotes were cultured in ultracentrifuged SOF + ITS + BSA to attain $3 \mathrm{~mL}$ of conditioned medium on Day 8. For each EV separation method, $1 \mathrm{~mL}$ of conditioned medium was used. A schematic representation of the DU, ODG, and SEC isolation protocols used in this study is shown in Figure 1. 


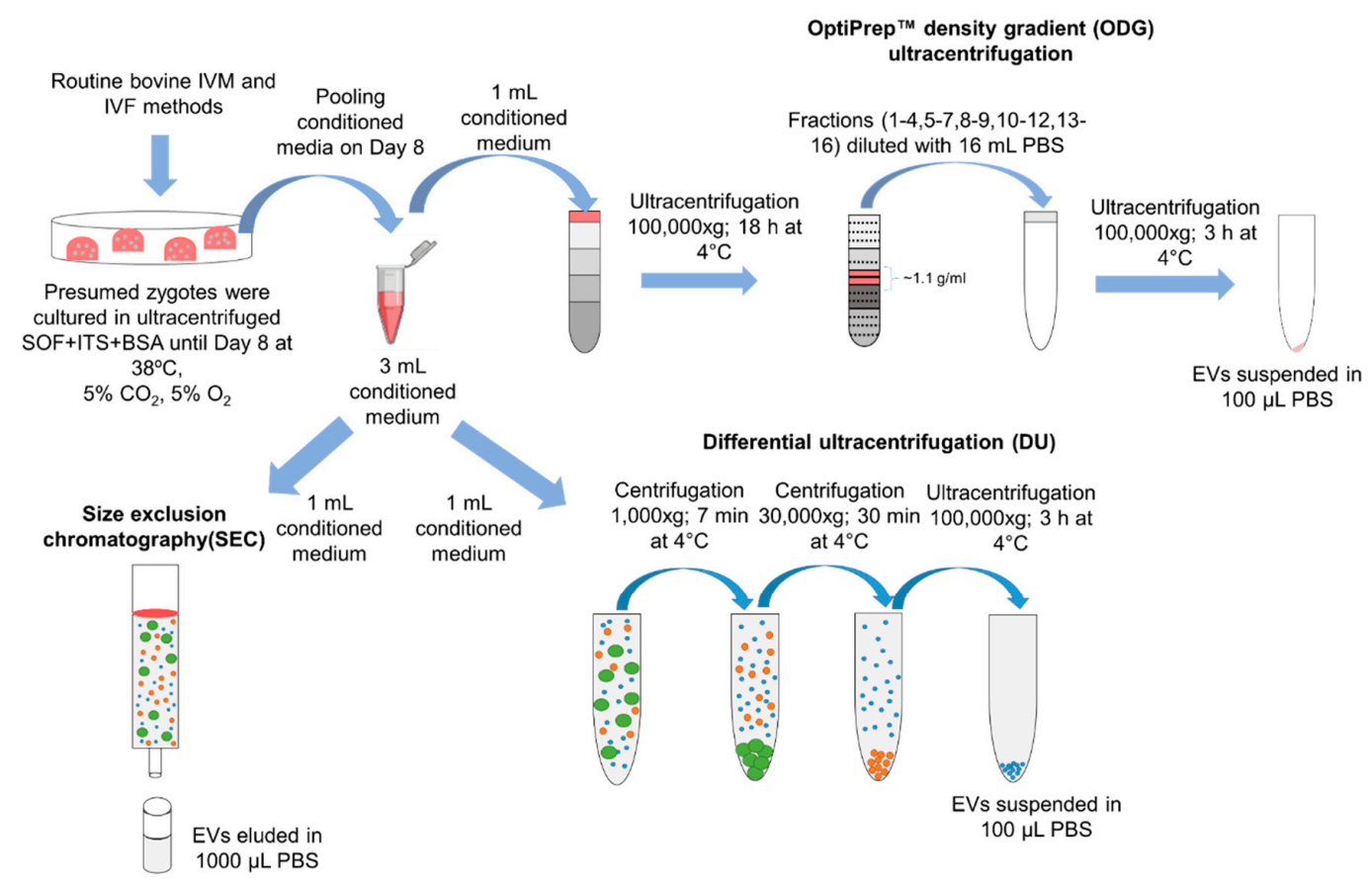

Figure 1. Schematic overview of three methods to separate EVs from bovine embryo-conditioned medium. A total of 1500 presumed zygotes were cultured in groups of 25 in $50 \mu \mathrm{L}$ droplets of ultracentrifuged synthetic oviductal fluid enriched with non-essential and essential amino acids (SOFaa), ITS ( $5 \mu \mathrm{g} / \mathrm{mL}$ insulin; $5 \mu \mathrm{g} / \mathrm{mL}$ transferrin; $5 \mathrm{ng} / \mathrm{mL}$ selenium) and medium droplets were covered with mineral oil and incubated at $38{ }^{\circ} \mathrm{C}$ in $5 \% \mathrm{CO}_{2}, 5 \% \mathrm{O}_{2}$, and $90 \% \mathrm{~N}_{2}$. After 8 days of post insemination, the bovine embryo-conditioned medium was pooled and equally divided over three separation methods: differential ultracentrifugation (DU), OptiPrep ${ }^{\mathrm{TM}}$ density gradient centrifugation (ODG), and size exclusion chromatography (SEC).

\subsection{Samples Separated by Differential Ultracentrifugation Contain Contaminants and Aggregates of Extracellular Vesicles}

Western blot analysis of EV preparations obtained by differential ultracentrifugation (DU) revealed the presence of EV-associated protein CD63 (Figure 2A). The DU pellet also contained high-density lipoprotein (HDL) marker apolipoprotein A-I (ApoA-I), and argonaute-2 (AGO-2) protein, a member of the RNA-induced silencing complex and a well-characterized extracellular RNA-binding protein (Figure 2A), indicating contamination of EVs with other extracellular particles. To cross-check the specificity of the Western blot bands, additional Western blotting was performed with incubation of secondary antibodies only (anti-rabbit IgG with 5\% BSA for CD63 and anti-mouse IgG with $5 \%$ milk for ApoA-I and AGO-2). We did not observe any non-specific bands with regards to the antibodies CD63 (42 kDa), AGO-2 (97 kDa), and ApoA-I (28 kDa), proving the specificity of the primary antibody (see Figure S1). TEM analysis confirmed the presence of EVs of approximately 100 to $260 \mathrm{~nm}$ in size (Figure 2B). In addition, TEM demonstrated the presence of protein aggregates and lipoprotein particles (Figure 2B). Next, we quantified the size and concentration of particles in DU preparations by NTA (Figure 2C). The particle concentration starting from a pool of 500 embryos was $3.02 \pm 0.36 \times 10^{10}$ particles $/ \mathrm{mL}$, and the mean and mode particle size were $266.8 \pm 2.1$ and $186.2 \pm 21.9 \mathrm{~nm}$, respectively (as detailed in Table 1, Figure 2C). 
A

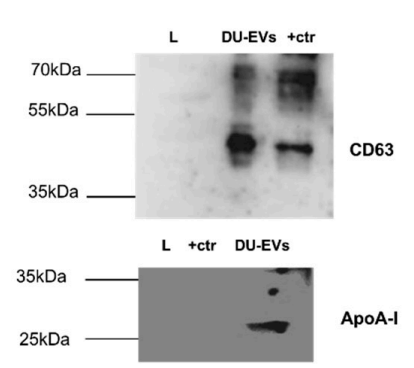

B

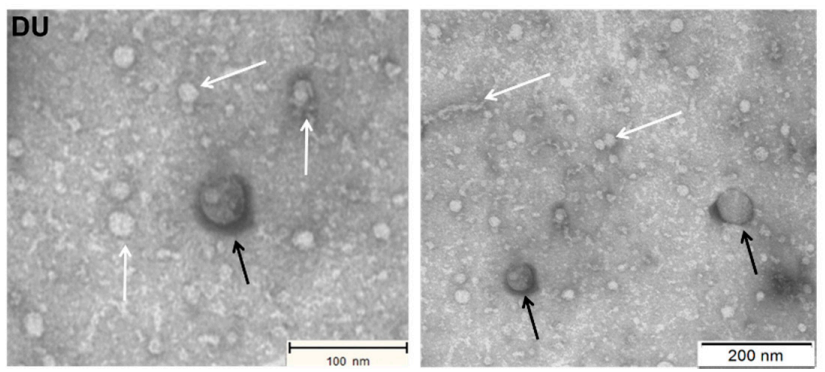

C

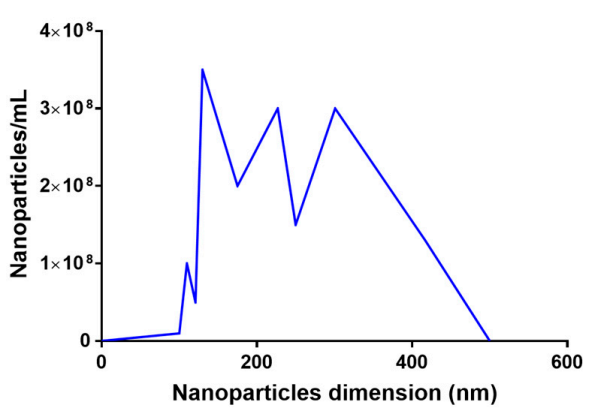

Figure 2. Characterization of DU-EVs derived from bovine embryo-conditioned medium. (A) Western blot analysis of EV-associated protein CD63 (42 kDa) and non-EV associated proteins (ApoA-I (28 kDa)). (B) Transmission electron microscopy pictures of DU-EVs with white arrows representing protein aggregates and black arrows indicating EVs. Scale bar $=100 / 200 \mathrm{~nm}$. (C) Size distribution profile of DU-EVs determined by NTA. Abbreviations: DU = differential ultracentrifugation; $+\mathrm{ctr}=$ follicular fluid derived EVs by OptiPrep ${ }^{\mathrm{TM}}$ density gradient; $\mathrm{L}=$ marker (protein ladder).

Table 1. Comparison of extracellular vesicle isolation methods by time, price, and nanoparticle analysis.

\begin{tabular}{|c|c|c|c|c|c|}
\hline $\begin{array}{l}\text { Isolation } \\
\text { Technique }\end{array}$ & DU & ODG & \multicolumn{3}{|c|}{ SEC } \\
\hline Working Principle & $\begin{array}{l}\text { EV isolation is based } \\
\text { on sequential } \\
\text { centrifugation steps to } \\
\text { exclude large vesicles } \\
\text { and cells debris and } \\
\text { precipitate EVs }\end{array}$ & $\begin{array}{l}\text { Combination of } \\
\text { ultracentrifugation } \\
\text { with sucrose } \\
\text { gradient }\end{array}$ & \multicolumn{3}{|c|}{$\begin{array}{c}\text { EV isolation is based on the size difference between EVs and } \\
\text { other particulate constituents }\end{array}$} \\
\hline Total time (h) & 5 & 22 & \multicolumn{3}{|c|}{2} \\
\hline $\begin{array}{c}\text { Approximate } \\
\text { price/sample }(€)\end{array}$ & 5 & 15 & \multicolumn{3}{|c|}{5} \\
\hline \multirow[t]{2}{*}{ Nanoparticle Data } & DU EVs & $\begin{array}{l}\text { OGD EVs } \\
\text { (8-9 Fraction) }\end{array}$ & \multicolumn{3}{|c|}{ SEC EVs Fractions } \\
\hline & & & SEC 9 & SEC 10 & SEC 11 \\
\hline Mean & $266.8 \pm 2.1 \mathrm{~nm}$ & $151.3 \pm 8.8 \mathrm{~nm}$ & $108.3 \pm 6.8 \mathrm{~nm}$ & $119.2 \pm 3.5 \mathrm{~nm}$ & $97.9 \pm 2.2 \mathrm{~nm}$ \\
\hline Mode & $186.1 \pm 21.9 \mathrm{~nm}$ & $100.1 \pm 20.4 \mathrm{~nm}$ & $102.3 \pm 7.0 \mathrm{~nm}$ & $99.7 \pm 6.0 \mathrm{~nm}$ & $68.4 \pm 4.0 \mathrm{~nm}$ \\
\hline $\mathrm{SD}$ & $91.2 \pm 2.8 \mathrm{~nm}$ & $53.9 \pm 26.3 \mathrm{~nm}$ & $34.9 \pm 4.3 \mathrm{~nm}$ & $52.4 \pm 6.9 \mathrm{~nm}$ & $39.4 \pm 3.3 \mathrm{~nm}$ \\
\hline D10 & $149.8 \pm 2.3 \mathrm{~nm}$ & $86.7 \pm 18.9 \mathrm{~nm}$ & $75.1 \pm 7.0 \mathrm{~nm}$ & $74.8 \pm 3.6 \mathrm{~nm}$ & $62.7 \pm 4.1 \mathrm{~nm}$ \\
\hline D50 & $264.5 \pm 5.3 \mathrm{~nm}$ & $127.9 \pm 6.5 \mathrm{~nm}$ & $101.1 \pm 7.7 \mathrm{~nm}$ & $104.3 \pm 3.8 \mathrm{~nm}$ & $86.4 \pm 4.4 \mathrm{~nm}$ \\
\hline D90 & $397.3 \pm 9.1 \mathrm{~nm}$ & $211.8 \pm 39.8 \mathrm{~nm}$ & $150.6 \pm 6.9 \mathrm{~nm}$ & $173.8 \pm 8.5 \mathrm{~nm}$ & $145.4 \pm 5.8 \mathrm{~nm}$ \\
\hline Nanoparticles/mL & $3.02 \pm 0.36 \times 10^{10}$ & $5.50 \pm 2.70 \times 10^{9}$ & $2.09 \pm 0.46 \times 10^{10}$ & $1.50 \pm 0.43 \times 10^{11}$ & $1.13 \pm 0.11 \times 10^{10}$ \\
\hline
\end{tabular}

Total time required (i.e., based on ease-of-use, turn-around time, hands-on time) and approximate cost per sample (i.e., based on the cost of centrifuge tubes and required solutions). The mode, mean value, standard deviation of size, and concentration for each EV isolation from 500 embryo culture medium method are provided. The value D50 represents the median size. Similarly, 90 percent of the distribution lies below the D90 value, and 10 percent of the population lies below the D10 value. 
2.2. Size Exclusion Chromatography does not Separate Protein Aggregates and Lipoprotein Particles from Extracellular Vesicles

Western blot analysis identified EV-associated protein CD63 across fractions 9 to 11. In addition, the majority of argonaute-2 (AGO-2) protein and apolipoprotein A-I (ApoA-I) was detected in fractions 9 to 11 (Figure 3A). Additional Western blotting analysis with secondary antibody (anti-rabbit IgG with 5\% BSA for CD63 and anti-mouse IgG with 5\% milk for ApoA-I, AGO-2) incubation showed some non-specific bands in the fractions of SEC 1 to 8 and SEC 10 (see Figure S2). No bands were observed with regards to ApoA-I (28 kDa), AGO-2 (97 kDa), and CD63 (42 kDa) from fractions 1 to 8 and fraction 10, proving the specificity of the primary antibody (Figure 3A). TEM analysis of fraction 10 confirmed the presence of EVs approximately 25 to $250 \mathrm{~nm}$ in size and protein aggregates (Figure 3B). SEC fraction 10 showed the highest particle concentration with $1.50 \pm 0.43 \times 10^{11}$ particles $/ \mathrm{mL}$ starting from a pool of 500 embryos with a mean particle size of $119.2 \pm 3.5 \mathrm{~nm}$ and a mode of $99.7 \pm 6.0 \mathrm{~nm}$ as measured by NTA (Table 1, Figure 3C).

\section{A}
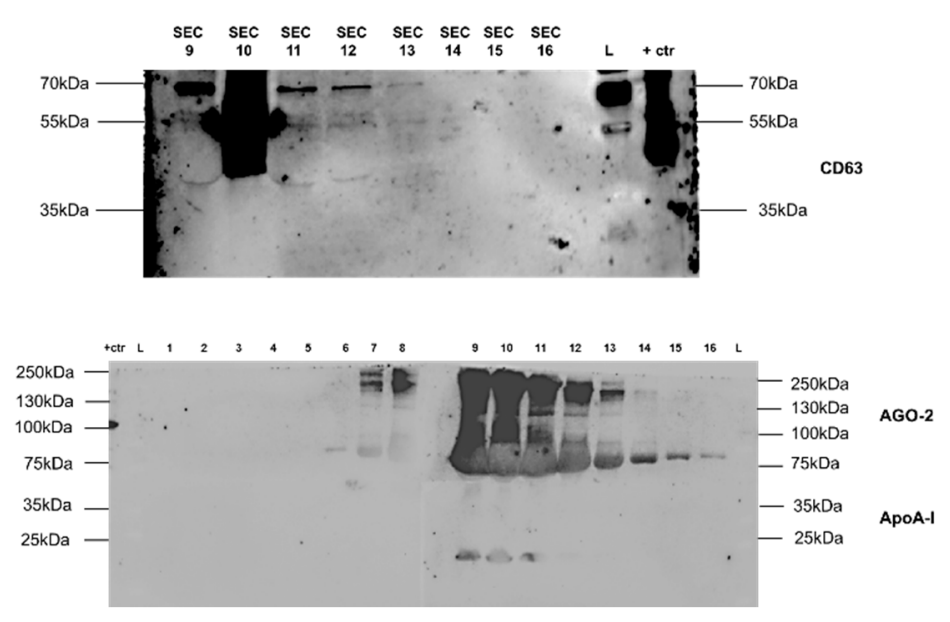

B
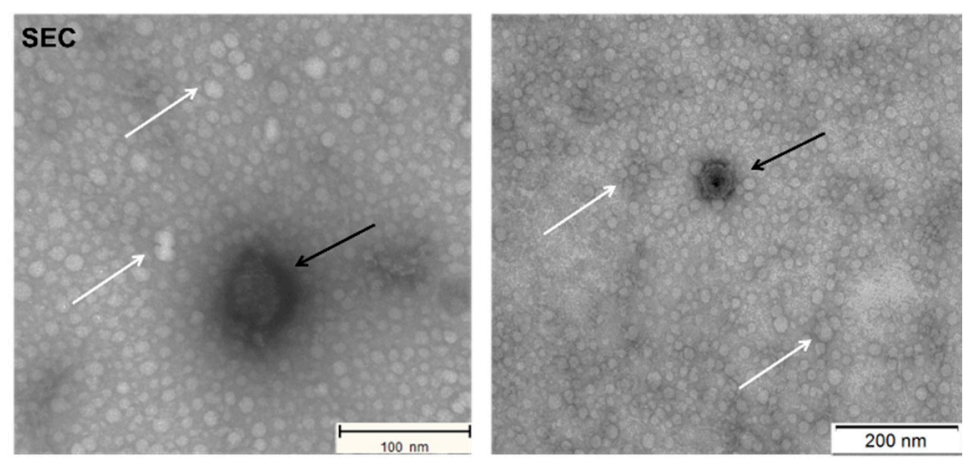

Figure 3. Cont. 


\section{C}

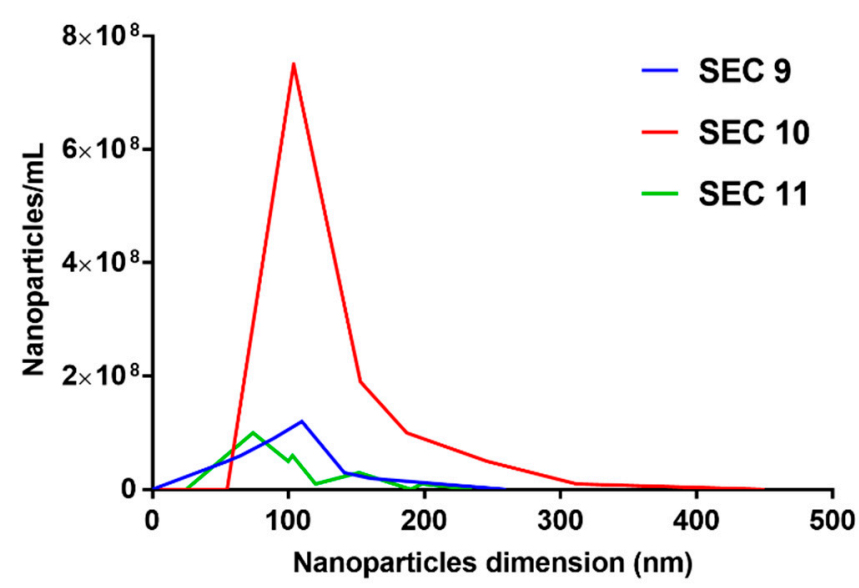

Figure 3. Characterization of SEC-EVs derived from bovine embryo-conditioned medium. (A) Western blot analysis of EV-associated protein CD63 (42 kDa) and non-EV associated proteins (ApoA-I (28 kDa), Ago-2 (97 kDa)). (B) Transmission electron microscopy pictures of SEC-EVs with white arrows representing protein aggregates and black arrows indicating EVs. Scale bar =100/200 nm. (C) Size distribution profile of SEC-EVs determined by NTA. Abbreviations: SEC $1=$ size exclusion chromatography fraction 1 (similar for 2 to 16 fractions); + ctr $=$ follicular fluid derived EVs by OptiPrep $^{\mathrm{TM}}$ density gradient; $\mathrm{L}=$ marker (protein ladder).

2.3. OptiPrep ${ }^{T M}$ Density Gradient Centrifugation Separates Extracellular Vesicles with Higher Specificity Compared to DU and SEC

Next, we analyzed the specificity of ODG centrifugation for enriching EVs from bovine embryo-conditioned medium. Western blot analysis identified EV-associated protein CD63 density fractions 8 and 9, corresponding to a density of $1.086-1.119 \mathrm{~g} / \mathrm{mL}$ (Figure $4 \mathrm{~A}$ ). By contrast, the majority of argonaute-2 (AGO-2) protein and apolipoprotein A-I (ApoA-I) were detected in, respectively, fractions 1 to 4 and 5 to 7 (density: 1.02-1.04, 1.20-1.23 g/mL, respectively; Figure $4 \mathrm{~A}$ ). There are some non-specific bands $(\sim 80 \mathrm{kDa})$ in the sample fraction of ODG 1-4 and a positive control sample of the secondary antibody Western blotting analysis (see Figure S1), whereas no additional bands were observed with regards to primary antibody incubation proving the specificity of AGO-2 antibody (Figure 4A). The TEM analysis of density fractions 8 and 9 confirmed the presence of EVs of approximately 25 to $250 \mathrm{~nm}$ in size (Figure 4B). The particle distribution assessed by NTA ranged from 25 to $300 \mathrm{~nm}$, with a mean particle size of $151.3 \pm 8.8 \mathrm{~nm}$, mode of $100.1 \pm 20.4 \mathrm{~nm}$, and a particle concentration of $5.50 \pm 2.70 \times 10^{9}$ particles $/ \mathrm{mL}$, starting from a pool of 500 embryos (Table 1, Figure $4 \mathrm{C}$ ). 
A
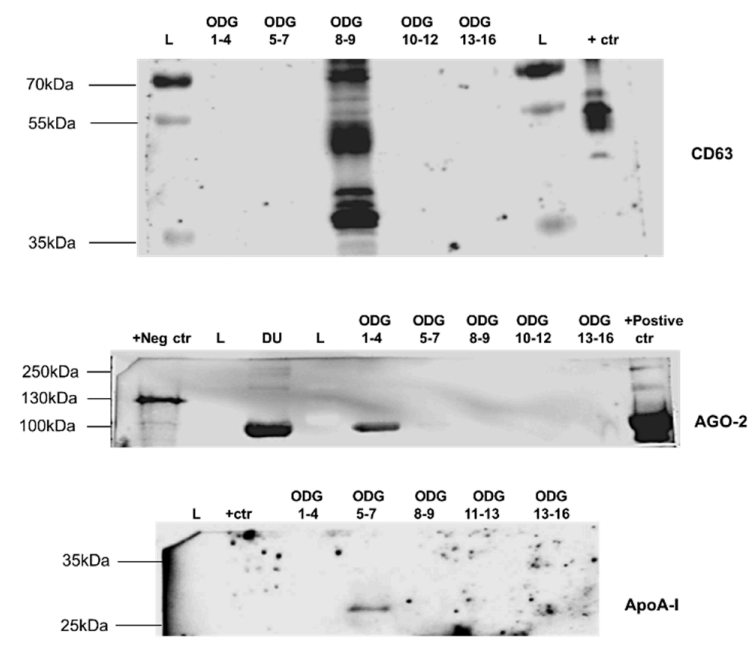

B
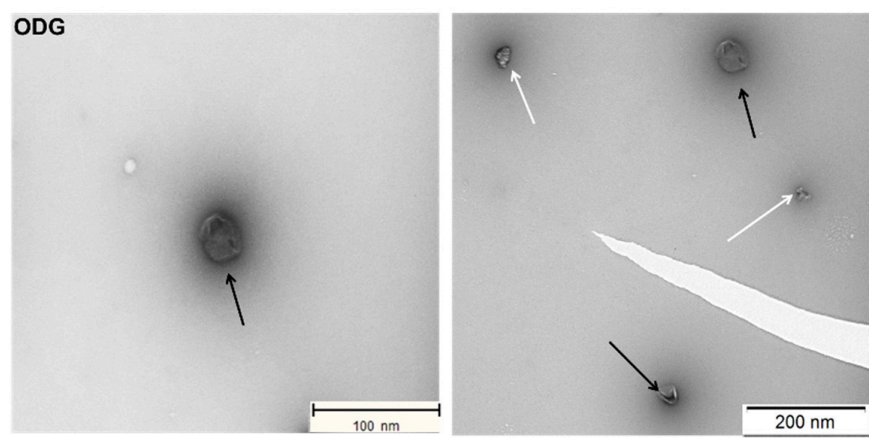

C

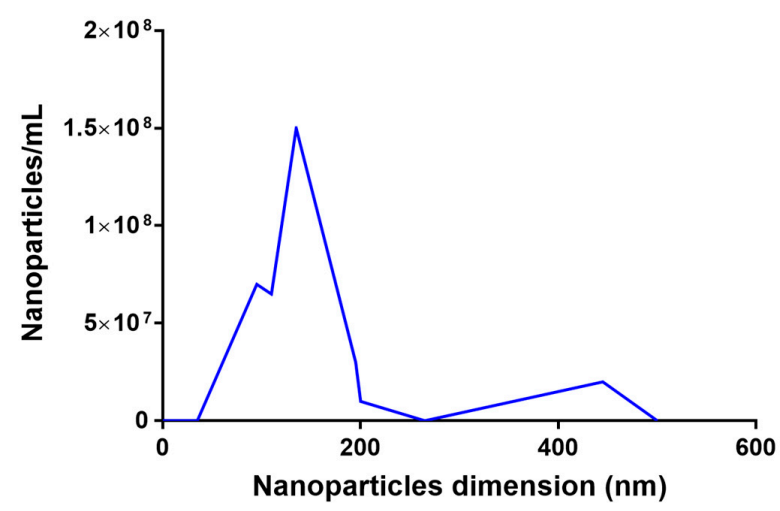

Figure 4. Characterization of ODG-EVs derived from bovine embryo-conditioned medium. (A) Western blot analysis of EV-associated protein CD63 (42 kDa) and non-EV associated proteins (ApoA-I (28 kDa), Ago-2 (97 kDa)). (B) Transmission electron microscopy pictures of ODG-EVs with white arrows representing protein aggregates and black arrows indicating EVs. Scale bar =100/200 nm. (C) Size distribution profile of ODG-EVs determined by NTA. Abbreviations: DU = differential ultracentrifugation; ODG 1-4 = OptiPrep $^{\mathrm{TM}}$ density gradient fractions 1 to 4 (similar for 5-7, 8-9. 10-12, 13-16); +ctr = follicular fluid derived EVs by ODG, L = marker (protein ladder) + Neg ctr = EVs derived from Lysate HEK 293T, +Positive ctr = Plasma pure. 


\section{Discussion}

There is a growing need for a standardized method to prepare EVs with sufficient specificity and efficiency. Multiple studies comparing different methods for separation of EVs from biological fluids and cell culture media have been published $[8,12,13]$. Such a comparison for medium conditioned by embryos has not been performed yet. Here, we assessed the efficiency of different EV separation methods, including DU, ODG centrifugation, and SEC. We show that ODG centrifugation separated EVs from bovine embryo-conditioned media with higher specificity compared to DU and SEC. As has been demonstrated by previous studies [8,14], ODG depletes EV samples from ribonucleoprotein complexes (argonaute-2) and lipoprotein particles (apolipoprotein A-I (ApoA-I)). In agreement with our findings, Van Deun et al. [8] also reported the presence of AGO-2 protein in EVs obtained by DU. In addition, DU may yield EV aggregates after pelleting [10,11,15,16]. Alternatively, it may enrich for larger EVs that sediment more efficiently. ODG centrifugation separates a wide range of smaller to larger EVs with high efficiency, confirming the results by Van Deun et al. [8].

When using ODG and SEC, the vesicle structure and integrity of EVs remain largely intact, and the biological activity of EVs is preserved. The size distribution profile of SEC- and ODG-derived EVs differed from DU-derived EVs, as SEC-and ODG-derived EVs were found to be smaller in size compared to DU-derived EVs. Larger size vesicles derived by DU may be the result of aggregation or fusion of EVs during ultracentrifugation, as also suggested by others $[10,17,18]$. The major disadvantages of using SEC methods are the co-precipitation of other extracellular particles like lipoprotein particles and ribonucleoproteins, as demonstrated per the current study. These findings are in accordance with Takov et al. $[19,20]$, who demonstrated that lipoproteins are co-isolated with SEC-EVs derived from plasma.

One of the major challenges in isolating EVs is the input volume of embryo culture medium. Earlier, Mellisho et al. [21] have demonstrated that $500 \mu \mathrm{L}$ of medium conditioned by bovine blastocysts was sufficient for EV isolation, but apparently, in this study, no standardized EV isolation method was applied. In our previous study, initially, we used $500 \mu \mathrm{L}$ of embryo-conditioned medium, but this was not sufficient for either characterization or quantification of EVs [6]. In conclusion, the separation of EVs with high specificity from $1 \mathrm{~mL}$ of embryo culture medium was addressed in the current study. Our analyses highlight the ODG centrifugation method as the most suitable procedure for EV enrichment that results in greater purity than DU and SEC. ODG centrifugation will allow the improved identification of EV-associated molecules that may further be used as biomarkers for embryo quality.

\section{Material and Methods}

\subsection{In Vitro Embryo Production}

Routine in vitro methods were used for bovine embryo production, as described previously by Pavani et al. [6]. Briefly, at the local abattoir, cow ovaries were obtained and processed within $2 \mathrm{~h}$ after collection. Upon arrival at the lab, ovaries were washed three times in warm physiological saline (with kanamycin $(25 \mathrm{mg} / \mathrm{mL}))$. Cumulus-oocyte complexes (COCs) were aspirated from antral follicles sizing between 4 and $8 \mathrm{~mm}$ diameter using an 18-gauge needle attached to a 10-mL syringe. Subsequently, only oocytes with uniformly granulated cytoplasm and surrounded by more than three compact layers of cumulus cells were cultured in groups of 60 COCs in $500 \mathrm{~mL}$ modified bicarbonate buffered TCM-199 (supplemented with $50 \mathrm{mg} / \mathrm{mL}$ gentamicin and $20 \mathrm{ng} / \mathrm{mL}$ epidermal growth factor) in $5 \% \mathrm{CO}_{2}$ in air for $22 \mathrm{~h}$ at $38.5^{\circ} \mathrm{C}$.

Frozen-thawed bull spermatozoa were separated using a 45/90\% Percoll ${ }^{\circledR}$ gradient (GE Healthcare Biosciences, Uppsala, Sweden). Next, the sperm pellet was washed in IVF-Tyrode's albumin-pyruvate-lactate (TALP) medium, containing bicarbonate-buffered Tyrode solution. Then, it was adjusted to a final concentration of $1 \times 10^{6}$ spermatozoa/mL using IVF-TALP medium enriched with BSA (Sigma A8806; $6 \mathrm{mg} / \mathrm{mL})$ and heparin $(25 \mathrm{mg} / \mathrm{mL})$. 
After $22 \mathrm{~h}$ maturation, bovine oocytes were washed in $500 \mu \mathrm{L}$ IVF-TALP and subsequently co-incubated with Percoll ${ }^{\circledR}$ washed bull spermatozoa. After $21 \mathrm{~h}$ gamete co-incubation, presumed zygotes were vortexed to remove surplus zona attached cumulus and sperm cells. The presumed zygotes were cultured in groups of 25 in $50 \mu \mathrm{L}$ droplets of ultracentrifuged synthetic oviductal fluid enriched with non-essential and essential amino acids (SOFaa), ITS (5 $\mu \mathrm{g} / \mathrm{mL}$ insulin; $5 \mu \mathrm{g} / \mathrm{mL}$ transferrin; $5 \mathrm{ng} / \mathrm{mL}$ selenium) and medium droplets were covered with mineral oil and incubated at $38^{\circ} \mathrm{C}$ in $5 \% \mathrm{CO}_{2}, 5 \% \mathrm{O}_{2}$, and $90 \% \mathrm{~N}_{2}$.

\subsection{Collection of Culture Media Conditioned by Bovine Embryos}

Culture medium conditioned by bovine embryos was collected and pooled in preparation for EV isolation. Per embryo culture replicate, a group of 100 presumed zygotes was cultured in SOF + ITS + BSA culture medium (UC BSA medium; 25 zygotes per $50 \mu \mathrm{L}$ droplet depleted from EVs after $100,000 \times g$ of ultracentrifugation for $18 \mathrm{~h}$ at $4{ }^{\circ} \mathrm{C}$ ). On $8 \mathrm{dpi}, 200 \mu \mathrm{L}$ embryo-conditioned medium was collected and stored at $-80^{\circ} \mathrm{C}$. To obtain $3 \mathrm{~mL}$ of embryo-conditioned culture medium, 15 replicates were performed with a total of 1500 presumed zygotes. One $\mathrm{mL}$ of pooled embryo-conditioned medium was used for each EV isolation method (differential ultracentrifugation, OptiPrep ${ }^{\mathrm{TM}}$ density gradient ultracentrifugation, and size-exclusion chromatography; as detailed in Figure 1).

\subsection{Differential Ultracentrifugation}

Differential ultracentrifugation was performed as described previously by Théry et al. [9] with a few modifications. Briefly, $1 \mathrm{~mL}$ of embryo-conditioned medium was diluted to $5 \mathrm{~mL}$ in phosphate-buffered saline (PBS) (Invitrogen), transferred to a 5.2-mL open-top polyallomer centrifuge tube (Beckman Coulter, Fullerton, CA, USA) and centrifuged for $7 \mathrm{~min}$ at $10,000 \times \mathrm{g}$ and $4{ }^{\circ} \mathrm{C}$ in a swinging bucket centrifuge (Optima XPN-80, SW 55 Ti rotor, Beckman Coulter). The pellet was discarded, and the supernatant was centrifuged for $30 \mathrm{~min}$ at $30,000 \times g$ and $4{ }^{\circ} \mathrm{C}$, again the pellet was discarded, and the supernatant was centrifuged for $3 \mathrm{~h}$ at $100,000 \times g$ and $4{ }^{\circ} \mathrm{C}$. The pellet was resuspended in $100 \mu \mathrm{L}$ of PBS and stored at $-80^{\circ} \mathrm{C}$ for further EV characterization.

\subsection{OptiPrep ${ }^{T M}$ Density Gradient Ultracentrifugation}

OptiPrep ${ }^{\mathrm{TM}}$ density gradient (ODG) ultracentrifugation was conducted, as previously reported by Van Deun et al. [8]. Briefly, appropriate amounts of a homogenization buffer (10 mM Tris- $\mathrm{HCl}$ (tromethamine-hydrochloric acid), $1 \mathrm{mM}$ EDTA (Ethylenediaminetetraacetic acid) and $0.25 \mathrm{M}$ sucrose ( $\mathrm{pH} 7.4)$ ) and an iodixanol working solution were mixed in order to prepare $5 \%, 10 \%, 20 \%$, and $40 \%$ iodixanol solutions. The iodixanol working solution was made by adding a working solution buffer (60 mM Tris-HCl, 6 mM EDTA, $0.25 \mathrm{M}$ sucrose (pH 7.4)) to a stock solution of OptiPrep ${ }^{\mathrm{TM}}(60 \%(w / v)$ aqueous iodixanol solution). Moreover, the gradient was prepared in a $16.8 \mathrm{~mL}$ open-top polyallomer tube (Beckman Coulter) by layering $4 \mathrm{~mL}$ of $40 \%, 4 \mathrm{~mL}$ of $20 \%, 4 \mathrm{~mL}$ of $10 \%$, and $3.5 \mathrm{~mL}$ of $5 \%$ solutions on top of each other. One $\mathrm{mL}$ of embryo-conditioned medium was overlaid onto the top of the gradient. Subsequently, the gradient was centrifuged at $4{ }^{\circ} \mathrm{C}$ for $18 \mathrm{~h}$ at $100,000 \times g$ (SW 32.1 Ti rotor, Beckman Coulter). Then, all 16 gradient fractions were divided into six samples by pooling the fractions $1-4$, $5-7,8-9,10-12,13-16$, respectively. The pooled fractions were added to $14 \mathrm{~mL}$ PBS. Subsequently, the separate suspensions were centrifuged at $4{ }^{\circ} \mathrm{C}$ for $3 \mathrm{~h}$ at $100,000 \times \mathrm{g}$. The resulting pellets were resuspended in $100 \mu \mathrm{L}$ PBS and stored at $-80^{\circ} \mathrm{C}$ for further EV characterization.

\subsection{Size-Exclusion Chromatography}

Sepharose CL-2B (GE Healthcare, Uppsala, Sweden) was washed three times with PBS containing $0.32 \%$ trisodium citrate dihydrate (ChemCruz, Dallas, TX, USA) [22,23]. For the preparation of one column, nylon net with $20 \mu \mathrm{m}$ pore size (NY2002500, Merck Millipore, Billerica, MA, USA) was placed on the bottom of a 10-mL syringe (BD Biosciences, San Jose, CA, USA), followed by stacking of $10 \mathrm{~mL}$ Sepharose CL-2B. On top of the SEC column, $1 \mathrm{~mL}$ of embryo-conditioned medium was loaded and 
fractions of 1-mL eluate were collected in 16 fractions. Resulting fractions were concentrated with Amicon Ultra-2 $10 \mathrm{k}$ centrifugal filters (UFC201024, Merck Millipore, Billerica, MA, USA) as previously described by Pavani et al. [6] and the eluates of each fraction were retrieved from the flow-through reservoir and stored at $-80{ }^{\circ} \mathrm{C}$ for further EV characterization.

\subsection{Characterization of Extracellular Vesicles}

\subsubsection{Western Blotting}

All samples were suspended in a reducing buffer $(0.005 \%$ bromophenol blue, $3 \% 2$-mercaptoethanol, $9.2 \%$ SDS, $40 \%$ glycerol, and $0.5 \mathrm{M} \mathrm{Tris-} \mathrm{HCl}(\mathrm{pH} 6.8)$ ) and boiled for $5 \mathrm{~min}$ at $95^{\circ} \mathrm{C}$. Protein samples were separated by SDS polyacrylamide gel electrophoresis and subsequently transferred to a nitrocellulose membrane (Bio-Rad, Hercules, California, USA). Next, the membrane was blocked at room temperature with 5\% skim milk/BSA TBST for $45 \mathrm{~min}$. Subsequently, the membranes were exposed to CD63 rabbit (1:200 in 5\% BSA + 0.5\% Tween PBS ab68418, Abcam, Cambridge, UK), AGO-2 rabbit (1:1000 in 5\% milk $+0.5 \%$ Tween PBS, ab32381, Abcam, Cambridge, UK), and ApoA-I mouse (1:1000 in 5\% milk $+0.5 \%$ Tween PBS, sc-376818, Santa Cruz, CA, USA), primary antibodies at $4{ }^{\circ} \mathrm{C}$. After overnight incubation, the membranes were extensively washed with $0.5 \%$ Tween in PBS. Then, the membranes were incubated with the appropriate secondary antibodies (anti-mouse IgG (GE Healthcare, UK), 1:3000 in 5\% milk + 0.5\% Tween PBS; anti-rabbit IgG (GE Healthcare, UK), 1:4000 in 5\% BSA + 0.5\% Tween PBS. After a final wash step, chemiluminescence substrate (WesternBright Sirius, Advansta, Menlo Park, California, USA) was added to the membranes. Imaging was performed using Proxima 2850 Imager (IsoGen Life Sciences, De Meern, The Netherlands).

\subsubsection{Transmission Electron Microscopy}

For transmission electron microscopy, each sample was deposited on precoated formvar/carbon support film copper mesh electron microscopy grids (FCF200H-CU-TB; Aurion, Netherlands). Grids were labeled for $45 \mathrm{~s}$ with $1 \%$ uranyl acetate (in double-distilled water). Prepared grids were examined using electron microscopy (JEM 1400 plus, JEOL, Benelux; and Ziess EM 109, Carl Zeiss, Jena, Germany). Moreover, images were made by Quemasa charge-coupled device camera (Olympus Soft Imaging solutions GMBH, Munster, Germany).

\subsubsection{Nanoparticle Tracking Analysis}

Samples were analyzed by nanoparticle tracking analysis (NTA) using the NanoSight LM10 microscope (Malvern Instruments Ltd., Malvern, UK) equipped with a 455-nm laser. For each individual sample, three videos of $60 \mathrm{~s}$ were recorded and analyzed with detection threshold 3 and camera level 13. All videos were analyzed by NTA Software version 3.2. To achieve optimal measurements, all EV samples were diluted with PBS to obtain a particle concentration within the optimal range $\left(3 \times 10^{8}-1 \times 10^{9}\right)$ of the NTA Software. Graphical presentations were performed using GraphPad Prism version 7.05 (GraphPad Software, San Diego, California, USA).

\subsubsection{EV-Track}

We have submitted all relevant data of our experiments to the EV-TRACK knowledgebase (EV-TRACK ID: EV190022; Consortium et al. [24] EV-TRACK: transparent reporting and centralizing knowledge in extracellular vesicle research). The EV-METRIC score is $78 \%$.

Supplementary Materials: Supplementary materials can be found at http://www.mdpi.com/1422-0067/21/8/2942/s1.

Author Contributions: K.C.P., A.H., A.V.S. and L.P. have designed the study. K.C.P. performed all experiments, analyzed the data, and wrote the manuscript. A.H., A.V.S. and L.P. helped in all experiments and discussed the draft manuscript. J.H., W.V.D.B. and L.C. helped with electron microscopy experiments. X.L. assisted in ovaries collection and IVF experiments. All authors reviewed and approved the final manuscript. 
Funding: This study was financed by European Union H2020 Marie Sklodowska-Curie (MSCA) Innovative Training Network (ITN) project Biology and Technology of Reproductive Health or REP-BIOTECH 675526 project.

Acknowledgments: The authors thank Petra Van Damme and Sofie De Geyter for their excellent technical assistance.

Conflicts of Interest: The authors declare no conflict of interest.

\section{References}

1. De Wever, O.; Hendrix, A. A supporting ecosystem to mature extracellular vesicles into clinical application. EMBO J. 2019, 38, e101412. [CrossRef] [PubMed]

2. Blank, C.; Duijf, I.T.; Slappendel, E.; Mischi, M.; Houterman, S.; Maas, J.W.M.; de Sutter, P.; Schoot, B.C. External validation of a prediction model to select the best day-three embryo for transfer in in vitro fertilization or intracytoplasmatic sperm injection procedures. Fertil. Steril. 2018, 110, 917-924. [CrossRef] [PubMed]

3. Popovic, M.; Dheedene, A.; Christodoulou, C.; Taelman, J.; Dhaenens, L.; Van Nieuwerburgh, F.; Deforce, D.; Van den Abbeel, E.; De Sutter, P.; Menten, B.; et al. Chromosomal mosaicism in human blastocysts: The ultimate challenge of preimplantation genetic testing? Hum. Reprod. 2018, 33, 1342-1354. [CrossRef]

4. Tšuiko, O.; Catteeuw, M.; Zamani Esteki, M.; Destouni, A.; Bogado Pascottini, O.; Besenfelder, U.; Havlicek, V.; Smits, K.; Kurg, A.; Salumets, A.; et al. Genome stability of bovine in vivo-conceived cleavage-stage embryos is higher compared to in vitro-produced embryos. Hum. Reprod. 2017, 32, 2348-2357. [CrossRef]

5. Stigliani, S.; Anserini, P.; Venturini, P.L.; Scaruffi, P. Exogen markers of human embryo quality in culture medium. Curr. Trends Clin. Embriol. 2015, 2, 209-221.

6. Pavani, K.C.; Hendrix, A.; Van Den Broeck, W.; Couck, L.; Szymanska, K.; Lin, X.; De Koster, J.; Van Soom, A.; Leemans, B. Isolation and Characterization of Functionally Active Extracellular Vesicles from Culture Medium Conditioned by Bovine Embryos In Vitro. Int. J. Mol. Sci. 2018, 20, 38. [CrossRef]

7. Giacomini, E.; Vago, R.; Sanchez, A.M.; Podini, P.; Zarovni, N.; Murdica, V.; Rizzo, R.; Bortolotti, D.; Candiani, M.; Viganò, P. Secretome of in vitro cultured human embryos contains extracellular vesicles that are uptaken by the maternal side. Sci. Rep. 2017, 7, 5210. [CrossRef]

8. Van Deun, J.; Mestdagh, P.; Sormunen, R.; Cocquyt, V.; Vermaelen, K.; Vandesompele, J.; Bracke, M.; De Wever, O.; Hendrix, A. The impact of disparate isolation methods for extracellular vesicles on downstream RNA profiling. J. Extracell. Vesicles 2014, 3. [CrossRef]

9. Théry, C.; Amigorena, S.; Raposo, G.; Clayton, A. Isolation and Characterization of Exosomes from Cell Culture Supernatants and Biological Fluids. Curr. Protoc. Cell Biol. 2006, 30, 3.22.1-3.22.29. [CrossRef]

10. Linares, R.; Tan, S.; Gounou, C.; Arraud, N.; Brisson, A.R. High-speed centrifugation induces aggregation of extracellular vesicles. J. Extracell. Vesicles 2015, 4. [CrossRef]

11. Bobrie, A.; Colombo, M.; Krumeich, S.; Raposo, G.; Théry, C. Diverse subpopulations of vesicles secreted by different intracellular mechanisms are present in exosome preparations obtained by differential ultracentrifugation. J. Extracell. Vesicles 2012, 1. [CrossRef] [PubMed]

12. Kalra, H.; Drummen, P.G.; Mathivanan, S. Focus on Extracellular Vesicles: Introducing the Next Small Big Thing. Int. J. Mol. Sci. 2016, 17, 170. [CrossRef] [PubMed]

13. Tauro, B.J.; Greening, D.W.; Mathias, R.A.; Ji, H.; Mathivanan, S.; Scott, A.M.; Simpson, R.J. Comparison of ultracentrifugation, density gradient separation, and immunoaffinity capture methods for isolating human colon cancer cell line LIM1863-derived exosomes. Methods 2012, 56, 293-304. [CrossRef]

14. Yuana, Y.; Levels, J.; Grootemaat, A.; Sturk, A.; Nieuwland, R. Co-isolation of extracellular vesicles and high-density lipoproteins using density gradient ultracentrifugation. J. Extracell. Vesicles 2014, 3. [CrossRef] [PubMed]

15. Mol, E.A.; Goumans, M.-J.; Doevendans, P.A.; Sluijter, J.P.G.; Vader, P. Higher functionality of extracellular vesicles isolated using size-exclusion chromatography compared to ultracentrifugation. Nanomed. Nanotechnol. Biol. Med. 2017, 13, 2061-2065. [CrossRef] [PubMed]

16. Lamparski, H.G.; Metha-Damani, A.; Yao, J.-Y.; Patel, S.; Hsu, D.-H.; Ruegg, C.; Le Pecq, J.-B. Production and characterization of clinical grade exosomes derived from dendritic cells. J. Immunol. Methods 2002, 270, 211-226. [CrossRef]

17. Taylor, D.D.; Shah, S. Methods of isolating extracellular vesicles impact down-stream analyses of their cargoes. Methods 2015, 87, 3-10. [CrossRef] 
18. Nordin, J.Z.; Lee, Y.; Vader, P.; Mäger, I.; Johansson, H.J.; Heusermann, W.; Wiklander, O.P.B.; Hällbrink, M.; Seow, Y.; Bultema, J.J.; et al. Ultrafiltration with size-exclusion liquid chromatography for high yield isolation of extracellular vesicles preserving intact biophysical and functional properties. Nanomed. Nanotechnol. Biol. Med. 2015, 11, 879-883. [CrossRef]

19. Takov, K.; Yellon, D.M.; Davidson, S.M. Confounding factors in vesicle uptake studies using fluorescent lipophilic membrane dyes. J. Extracell. Vesicles 2017, 6, 1388731. [CrossRef]

20. Takov, K.; Yellon, D.M.; Davidson, S.M. Comparison of small extracellular vesicles isolated from plasma by ultracentrifugation or size-exclusion chromatography: Yield, purity and functional potential. J. Extracell. Vesicles 2018, 8, 1560809. [CrossRef]

21. Mellisho, E.A.; Velásquez, A.E.; Nuñez, M.J.; Cabezas, J.G.; Cueto, J.A.; Fader, C.; Castro, F.O.; Rodríguez-Álvarez, L. Identification and characteristics of extracellular vesicles from bovine blastocysts produced in vitro. PLoS ONE 2017, 12, e0178306. [CrossRef] [PubMed]

22. Vergauwen, G.; Dhondt, B.; Van Deun, J.; De Smedt, E.; Berx, G.; Timmerman, E.; Gevaert, K.; Miinalainen, I.; Cocquyt, V.; Braems, G.; et al. Confounding factors of ultrafiltration and protein analysis in extracellular vesicle research. Sci. Rep. 2017, 7, 2704. [CrossRef] [PubMed]

23. Tulkens, J.; Vergauwen, G.; Van Deun, J.; Geeurickx, E.; Dhondt, B.; Lippens, L.; De Scheerder, M.-A.; Miinalainen, I.; Rappu, P.; De Geest, B.G.; et al. Increased levels of systemic LPS-positive bacterial extracellular vesicles in patients with intestinal barrier dysfunction. Gut 2018. [CrossRef] [PubMed]

24. Consortium, E.-T.; Van Deun, J.; Mestdagh, P.; Agostinis, P.; Akay, Ö.; Anand, S.; Anckaert, J.; Martinez, Z.A.; Baetens, T.; Beghein, E.; et al. EV-TRACK: Transparent reporting and centralizing knowledge in extracellular vesicle research. Nat. Methods 2017, 14, 228.

(C) 2020 by the authors. Licensee MDPI, Basel, Switzerland. This article is an open access article distributed under the terms and conditions of the Creative Commons Attribution (CC BY) license (http://creativecommons.org/licenses/by/4.0/). 